\title{
IP OVER ATM - PERFORMANCE EVALUATION, EXPERIMENTS AND RESULTS
}

\author{
Paulo C. A. Antunes, Walter Godoy Júnior, Eduardo Nisenbaum \\ Centro Federal de Educação Tecnológica do Paraná - Centro de Pós Graduação em \\ Engenharia Elétrica e Informática Industrial - Curitiba - Paraná - Brazil \\ E-mail (pcaa, godoy, baum\} @cpgei.cefetpr.br
}

\begin{abstract}
This paper evaluates the performance of the IP protocol having ATM technology as the network support. Two models are approached: "The Classical IP and ARP over ATM" and "LAN Emulation". The theoretical performance was calculated based on the protocol overhead sources and compared to practical experiments. It is shown that: for applications working in IP over ATM environment, when we use short blocks of information transfer, the performance deteriorates considerably; and when we increase its size the performance, on the average, is limited about $86 \%$ of the line speed capacity. The influence of MTU size of the IP layer in the performance is also analyzed.
\end{abstract}

Keywords: ATM, IP, Performance

\section{INTRODUCTION}

The Asynchronous Transfer Mode (ATM) is a technology projected to integrate voice, video and computer data services, supplying individually to each service and application the Quality of Service (QoS) requested. The ATM is the connection-oriented service that uses small units of fixed size, denominated cells, in the transport of information, that allows fast hardware switching [1].

Due to wide use of the IP (Internet Protocol), dominant in the interconnection of networks and that presents continuous expansion in the world network (Internet), we will analyze the ATM as network support for the IP Protocol. To study objectifying the impacts of performance that this integration presents, in this paper we analyzed the proposed models by IETF "The Classical IP and ARP over ATM"[2] and by ATM Forum "LAN 
Emulation"[3]. In both models the ATM is seen like a Data Link Layer, in the same way that it happens with Ethernet, Token Ring, FDDI, FrameRelay, X.25, etc. The IP when uses ATM needs to adapt its packets, of variable data size, through the protocol in the ATM Adaptation Layer (AAL5) into cells fixed size small. However such procedure imposes overhead and processing cost. Such characteristics are focalized in this work.

This paper is organized in six sections. The second refers to the topology and equipments used in the experiment. In the third section we analyze performance limiting factors in the integration of the IP protocol environment ATM. In the fourth the proposed models by IETF and ATM Forum are analyzed and their overhead sources quantified. In the fifth section the realized experiments are shown and finally in the sixth the conclusions are written.

\section{TOPOLOGY AND EQUIPMENT OF THE NETWORK}

The tests were made at the laboratories of the high-speed network of Curitiba, Brazil, specially in the CEFET (Centro Federal de Educação Tecnológica do Paraná) and UFPR(Universidade Federal do Paraná) institutions (connected through 6.200 meters of single-mode optical fibers). The network topology is shown in Figure 1.

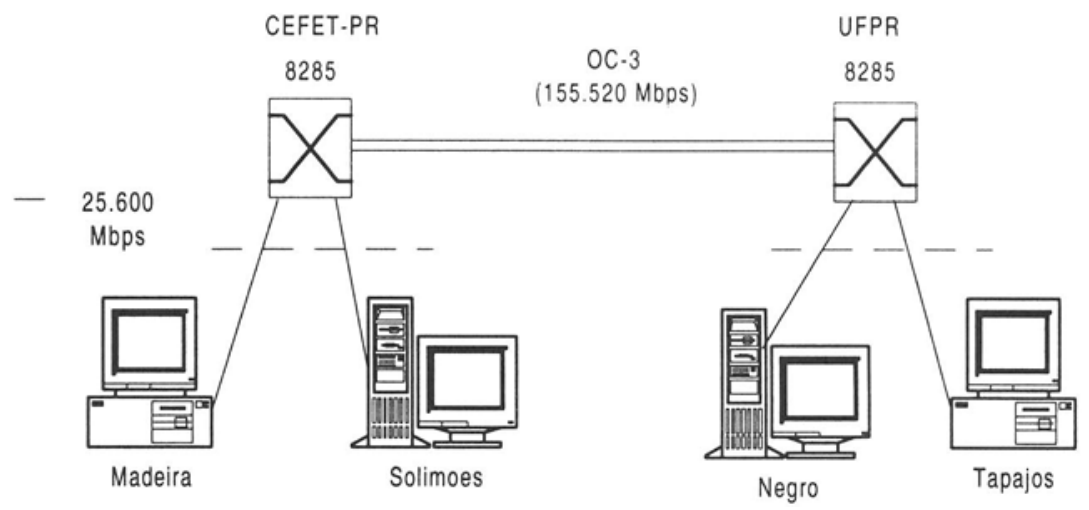

Figure 1: Network Topology

Switches ATM (IBM-8285) were used with backplane of 4.2 Gbps and it use the technique of EPD(Early Packet Discard)[4]. EPD is the technique, where in case of congestion, instead of discarding aleatory cells of each packet, all the cells belonging to the same packet but last are discarded. This 
avoids the unnecessary sending of cells that cannot be used in the receptor. The network interfaces used have PCI (Peripheral Component Interconnect) architecture with nominal capacity of $25.600 \mathrm{Mbps}$. The stations, CPU, operating systems and network interfaces used are described in Table 1.

Table 1: Stations, processors, operating systems and network adapters used

\begin{tabular}{|l|l|l|}
\hline Station & \multicolumn{1}{|c|}{$\begin{array}{c}\text { Processor and Operating } \\
\text { System }\end{array}$} & $\begin{array}{c}\text { Network } \\
\text { Adapter }\end{array}$ \\
\hline Solimoes & Power PC 233 MHz, AIX 4.2.1 & Turboways \\
\hline Negro & Power PC 233 MHz, AIX 4.2.1 & Turboways \\
\hline Madeira & IBM-PC Pentium 166 MHz, NT 4.0 & Turboways \\
\hline Tapajos & IBM-PC Pentium 166 MHz, Linux & ForeRunner \\
\hline
\end{tabular}

In the physical interface local connection, between the stations and the switch, Unshielded Twisted Pairs UTP cabling (category 5) were used. In the remote connection of the Metropolitan Area Network (MAN), the switches were interconnected by optical link with single-mode fibers, with line rate of $155.520 \mathrm{Mbps}$. In the physic layer, the interface of 25.600 Mbps[5][6] doesn't have frame overhead, cells are transmitted in direct flow and the separation among them is done by the HEC field (Header Error Control) contained in each ATM cell[7]. The physical interfaces between switches were configured to use SONET (Synchronous Optical Network) frames. Figure 2 [7] shows a SONET frame composed of three STS1 signals connected forming a STS-3c/OC-3c signal. Each SONET frame repeats itself to each $125 \mu \mathrm{s}(8000$ frames a second $)=8000 \times[9 \times(261+9)]$ $\mathrm{x} 8$ bits], supplying the transmission rate of $155.520 \mathrm{Mbps}$.

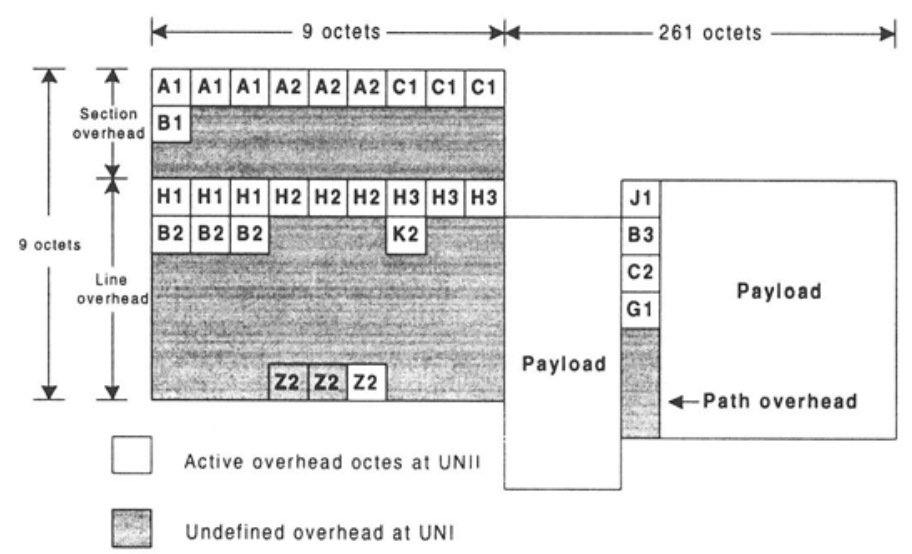

Figure 2: SONET frame STS-3C/OC-3C 
The total size of the SONET STS-3c/OC-3c frame is 2430 octets $[9 \times(9+261)]$. Of which 90 octets are of overhead, divided in:

- The section overhead $=27$ octets;

- The line overhead $=54$ octets;

- The path overhead $=9$ octets.

\section{PERFORMANCE LIMITING FACTORS}

The application, when transfers data to the network, involves the interaction of protocols in several layers. Each protocol can insert control informations to those data to identify it, in the header form, trailer or both. These information increase the reliability in the transport of the data, for instance, with the use of the fields: HEC (Header Error Control) of the ATM cell, CRC (Cyclic Redundancy Check) of AAL5, sum of integrity of the data (checksum) in the IP protocols, TCP (Transmission Control Protocol) and UDP (User Datagram Protocol). The information also identify the partners of the communication, as in the following case: in ATM the VPI (Virtual Path Identifier) and VCI (Virtual Channel Identifier); in IP the source and destination address; in TCP and UDP the indication of the communication port and PDU (Protocol Data Unit) size. These additional informations are denominated protocol overhead and it is one of the factors that limit the application to use at wire speed.

\section{MODELS OF CLASSICAL IP AND LANE}

The IP protocol in the Classical IP and LANE models use the protocol of the adaptation layer AAL5. The allowed maximum size of PDU(Protocol Data Unit) in CS(Convergence Sublayer) is of 65535 octets[8]. The format of the frames of the AAL5 and ATM layers is shown in Figure 3, where CPCS is the Common Part Convergence Sublayer and SAR is the Segmentation and Reassembly Sublayer.

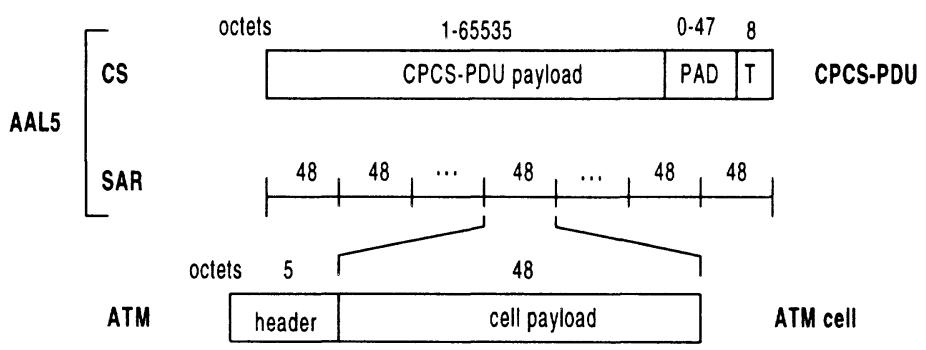

Figure 3: Frames of the AAL5 and ATM layers 
In the transmission the SAR segments the PDU in blocks of 48 octets, no protocol identification is inserted in this sublayer. The SAR delivers the blocks, now denominated ATM-SDU(ATM-Service Data Unit) to the ATM layer and through the user-to-user indication parameter of primitive ATMDATA.indication, informs the ATM layer which is the last block of the frame. The ATM layer inserts the header in each PDU, now denominated cell, marks the last unit of the frame, and delivers the physical layer for transmission. In the reception the SAR extracts the payload of the cells, convert them again in PDU and delivers to the superior protocols.

\subsection{Classical IP}

The default value of MTU (Maximum Transmission Unit) for the IP protocol in the layer AAL5 is of 9180 octets[2]. In the experiments beyond of that value, MTU of 1500 octets were used, too. The MTU indicates the maximum amount of data that can be inserted in one PDU without fragmentation. Figure 4 shows the format of the PDUs in the layers of the transport protocols TCP and UDP for the model of Classical IP and ARP over ATM, as well as the amount of overhead and the number of corresponding octets and cells.

Each TCP or UDP segment has direct relationship with each IP packet, however the transport protocol can include several blocks of data in each segment[9].

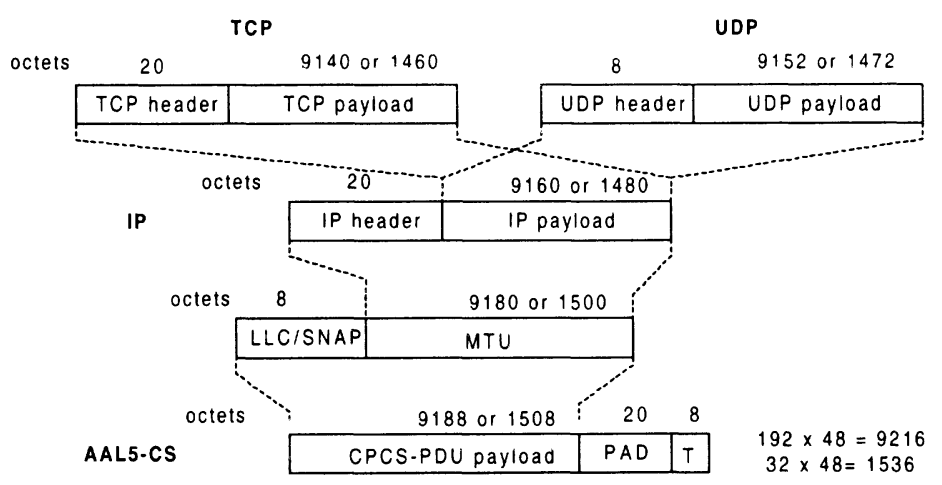

Figure 4: PDU format for the TCP,UDP, IP over AAL5 in Classical IP Model

\subsection{LANE}

The LAN Emulation (LANE) transports frames of the traditional Ethernet and Token-Ring networks; we concentrated experiments on Ethernet 
LANES. The IEEE-802.3[10] standard for Ethernet networks defines that the minimum size of a frame should be of 64 octets, with 46 payload octets and 18 overhead octets. This standard also defines that the maximum size of the frame should be of 1518 octets, 1500 are payload octets and the rest, 18 octets, identify the frame.

When transporting Ethernet frames, the LANE excludes the field of CRC, because it is made in CPCS-PDU. The LANE model adds in each Ethernet frame one header of 2 octets. This header contains the LAN Emulation Client Identifier (LECID).

In the AAL5 adaptation protocol a trailer of 8 octets is added to each frame and, if necessary, it adds octets of padding (PAD) to turn the CPCSPDU size a multiple of 48 octets ( that are delivered to SAR). The trailer is only present in the last cell of each PDU. The recommendation of ITU-T[8] defines that the content of the trailer has to be constituted by fields CPCSUU, used to transfer user to user informations of CPCS, of the Common Part Indicator(CPI) of CPCS, of the field length of CPCS-PDU, as well as of CRC that check the whole CPCS-PDU. The CPI field is still not defined, however actually it is used to turn the trailer to the size of 8 octets. Figure 5 shows the trailer format of the CPCS-PDU.

\begin{tabular}{|c|c|c|c|}
\hline octets & 1 & 2 & 4 \\
\hline CPCS.UU & CPI & Length & CRC \\
\hline
\end{tabular}

Figure 5: Trailer format to CPCS-PDU

The default size of a frame in AAL5-SDU in LANE is 1516 octets. This generates a PDU of 1536 octets in Ethernet, in which 1516 octets are data and the remaining 20 octets are overhead. One AAL5-SDU frame of 1536 octets corresponds to 32 cells in the ATM layer. The recommendation of ATM Forum[3] also allows to use larger sizes of AAL5-SDU(4544, 9234 and 18190) octets. In the experiment with standard size of Ethernet frames we used 1536 octets. Figure 6 shows the format of the PDU on TCP, UDP layer for the LANE model using AAL5, as well as the overhead distribution in the layers and the number corresponding on octets and cells. 


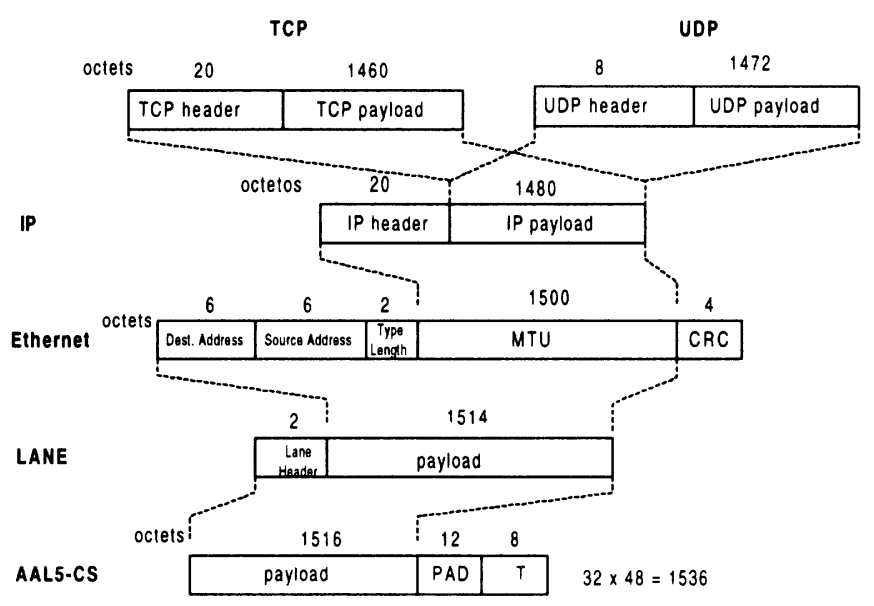

Figure 6: PDU format to TCP, UDP, IP, Ethernet over AAL5 in LANE

\subsection{Theoretical Calculations of the Available Maximum Bandwidth for the Model of Classical IP}

In order to evaluate the results obtained experimentally, initially the theoretical available maximum bandwidth was calculated to the application involving all the protocol layers. In the calculations were considered just the imposed overhead by the data unit of protocol in each layer.

Through the expressions below we calculated the available bandwidth(BW) and Table 2 shows the calculated values for:

- ATM layer between switches

$$
\mathrm{BW}_{\mathrm{ATM}}=\frac{\text { frame_payload }_{\text {frame_length }}}{\text { frit }_{-} \text {rate }}
$$

- ATM adaptation layer

$$
\mathrm{BW}_{\mathrm{AAL}}=\frac{\text { cell__payload }}{\text { cell_legth }} \cdot \mathrm{BW}_{\mathrm{ATM}}
$$

- IP

$$
\mathrm{BW}_{\mathrm{IP}}=\frac{\mathrm{IP} \_\mathrm{MTU}}{\mathrm{CPCS} \_\mathrm{PDU}} \cdot \mathrm{BW} \text { AAL }
$$

- TCP and UDP

$$
B W(T C P)=B W(U D P) \cdot \frac{I P_{-} \text {payload }}{I P_{-} M T U} \cdot B W I P
$$

- Application when TCP is used 
$\mathrm{BW}_{\text {app }}=\frac{\mathrm{TCP}_{\text {_payload }}}{\mathrm{IP} \text { _payload }} \cdot \mathrm{BW}_{\mathrm{TCP}}$

- Application when UDP is used $\mathrm{BW}_{\mathrm{appl}}=\frac{\mathrm{UDP}_{\text {p payload }}}{\mathrm{IP}_{\text {_payload }}} \cdot \mathrm{BWUDP}$

Table 2: Bandwidth available after protocol overhead

\begin{tabular}{|c|c|c|c|c|c|}
\hline (Mbps) & \multicolumn{4}{|c|}{ SONET OC-3c } & UTP -25.600 \\
\hline Line rate & \multicolumn{4}{|c|}{155.520} & 25.600 \\
\hline To ATM & \multicolumn{4}{|c|}{149.760} & 25.600 \\
\hline \multirow[t]{3}{*}{ To AAL } & \multicolumn{4}{|c|}{135.632} & 23.185 \\
\hline & \multicolumn{4}{|c|}{$\begin{array}{l}\text { Classical IP } \\
\text { MTU(octets) }\end{array}$} & $\begin{array}{c}\text { LANE } \\
\text { MTU(octets) }\end{array}$ \\
\hline & \multicolumn{2}{|c|}{9180} & \multicolumn{2}{|c|}{1500} & 1500 \\
\hline To IP & \multicolumn{2}{|c|}{23.094} & \multicolumn{2}{|c|}{22.642} & 22.642 \\
\hline To TCP & \multicolumn{2}{|c|}{23.044} & \multicolumn{2}{|c|}{22.340} & 22.340 \\
\hline To UDP & \multicolumn{2}{|c|}{23.044} & \multicolumn{2}{|c|}{22.340} & 22.340 \\
\hline & TCP & UDP & TCP & UDP & TCP UDP \\
\hline To Appl. & 22.994 & 23.024 & 22.038 & 22.219 & 22.038 \\
\hline
\end{tabular}

\section{TRAFFIC MEASUREMENTS}

In the measurements the program called Netperf developed by the Hewlett-Packard Company [11] was used. It uses the client/server architecture and utilizes socket in the communication among processes and between machines and processes. The tests consisted of sending continually prefixed size data blocks. To obtain index of reliability of $95 \%( \pm 1,5 \%)$, periods of 60 seconds were necessary for each individual variation of parameter.

\subsection{Local Test of Performance}

The local test consisted of sending and receiving flows of tabulated data in the same communication port and in the same station. To estimate the station capacity of processing interacting with the protocol stack, when the TCP transport protocol is used, in agreement with the recommendation in [12] duplicates the value obtained in the transmission. Table 3 shows the results obtained in each station for the TCP. The stations were configured with the following parameters: MTU of 1500 octets in the IP protocol; 
maximum SDU of 1516 octets in AAL5 and socket buffers for transmission and reception using the same sizes $=4096$ octets.

Table 3: Results of the obtained tests using the TCP protocol

\begin{tabular}{|l|c|c|}
\hline \multicolumn{1}{|c|}{ Station } & $\mathbf{T X}=\mathbf{R X}(\mathbf{M b p s})$ & Estimated capacity (Mbps) \\
\hline Solimoes & 115.567 & 231.124 \\
\hline Negro & 114.231 & 228.462 \\
\hline Madeira & 41.480 & 82.960 \\
\hline Tapajos & 111.782 & 223.564 \\
\hline
\end{tabular}

\subsection{Tests Among Stations}

In the practical tests we analyzed the IP using the TCP transport protocol and the measures were collected in the transmitter. The environment was controlled, there wasn't competition for bandwidth for the analyzed models used UBR service (Unspecified Bit Rate) and in case of congestion they have preference in the discard.

\subsection{Classical IP}

Figure 7 shows the results obtained among the Solimoes and Negro stations; aimed at to evaluate the influence of socket buffer size in relation to variation of the size of the messages when MTU of 1500 octets is used. Figure 8 is analogous to Figure 7, however MTU of 9180 octets was used. In both cases the socket buffers of transmission and reception were configured with the same sizes, beginning with 4096 octets and in the sequence increased by multiples of this value until 65536 octets. The messages began with 16 octets and were increased by the factor 4 in relation to the previous value and concluded in 65536 octets. 


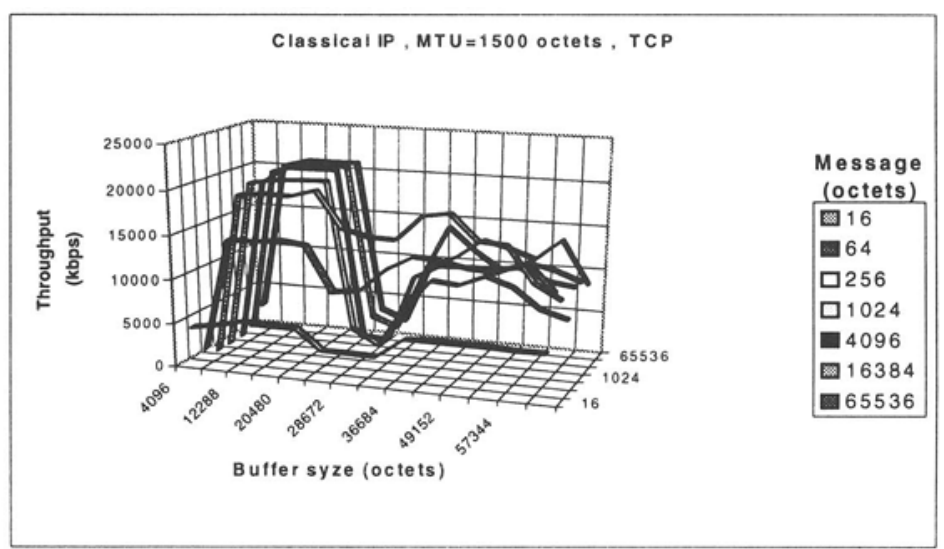

Figure 7: Throughput vs. socket buffer size vs. message size

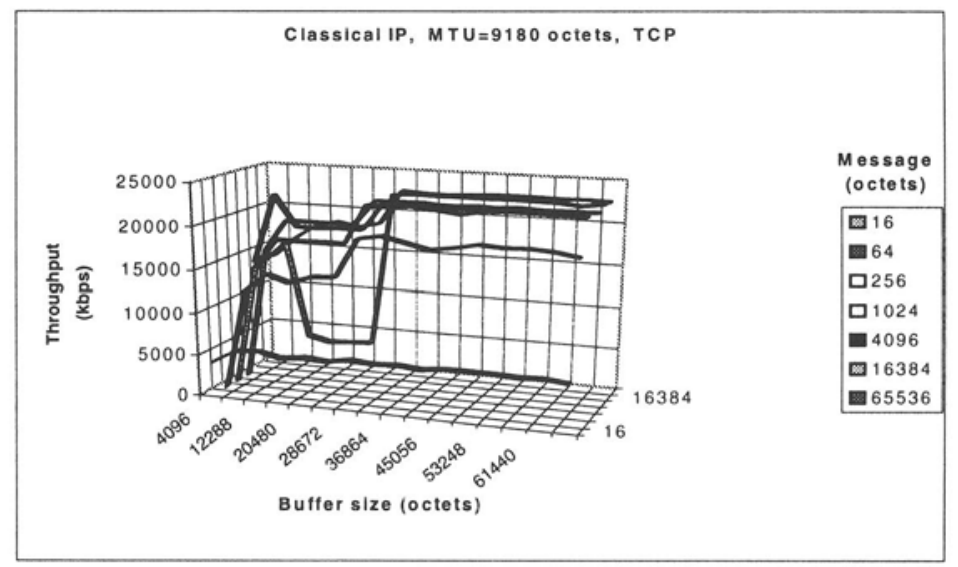

Figure 8: Throughput vs. socket buffer size vs. message size

It is specially observed that messages of small size (16 and 64 octets) in both cases presented a low performance, this is due to the overhead octets of the protocol layers being larger than the message size, still allied to the countless interruptions generated by the physical interface and to system calls, at the level of operating system. In the other messages the best found performance went to socket buffer size among 8192 octets and 16384 octets when it uses MTU(IP) of 1500 octets. To MTU 9180 octets the best performance went to socket buffers size larger of 28672 octets..

The result are shown in Figure 9 and 10 involving the stations with smaller processing capacity, Madeira and Tapajos respectively. In them messages were tabulated with multiple size of the IP MTU default and socket buffers with multiple size of those values. 


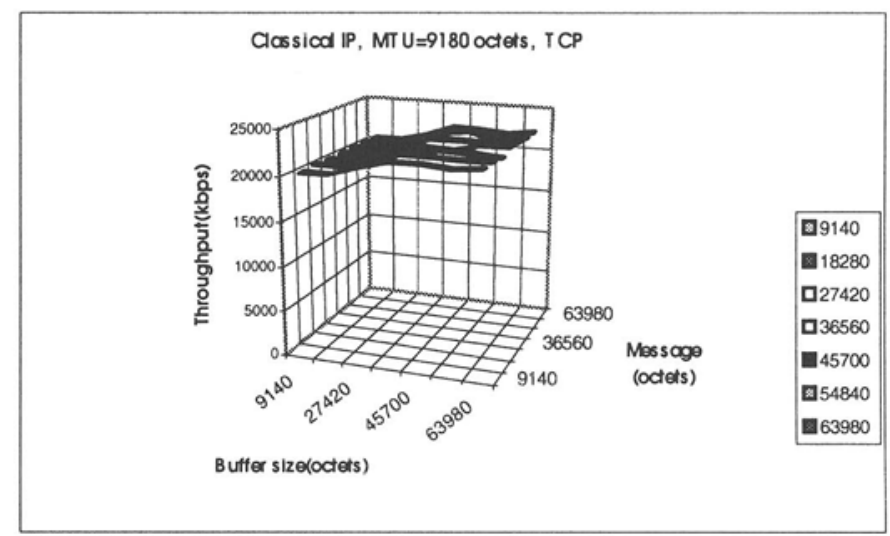

Figure 9: Experiment between the Madeira and Tapajos stations

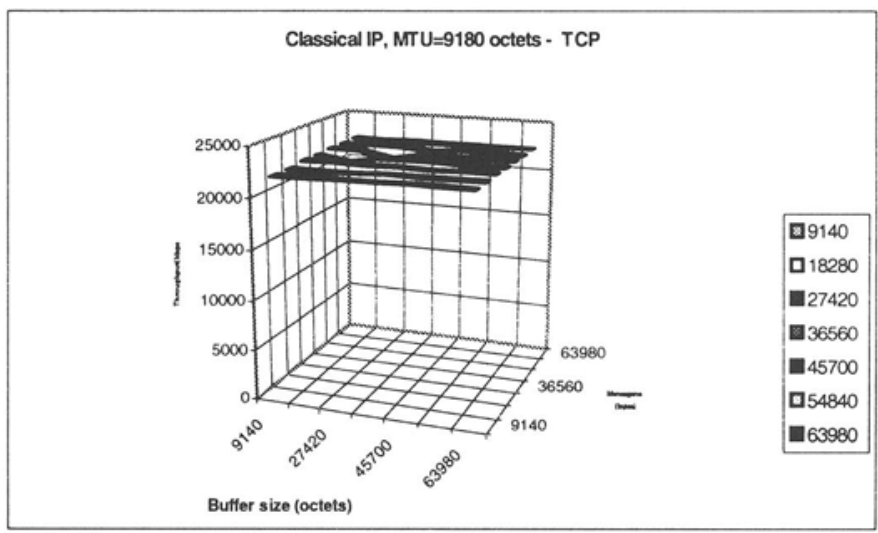

Figure 10: Experiment between the Tapajos and Solimoes stations

As the Tapajos as the Madeira stations demonstrated similar performances, obtaining maximum performance of $22.420 \mathrm{Mbps}$ and $22.320 \mathrm{Mbps}$ respectively that correspond to $97 \%$ of the calculated capacity.

\subsection{LANE}

In the experiments with LANE were pre-fixed socket buffer sizes in 16384 octets and messages beginning with 16 octets, leaving for 64 octets and proceeding for multiples of this value to reach 4096 octets. As it can be observed by Figures 11 and 12 , the performance was considerably reduced for messages of small size, analogous to what was found in Classical IP model. 


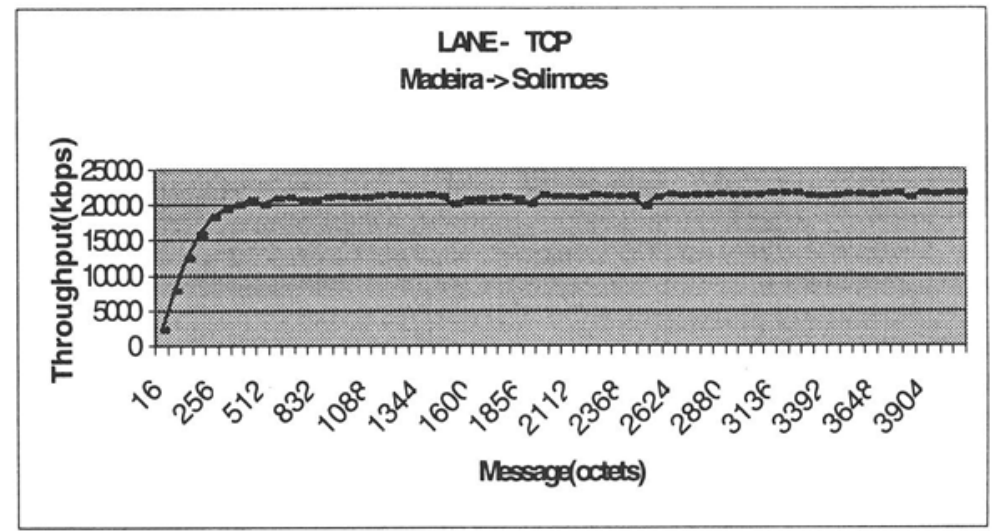

Figure 11: Throughput vs. message size

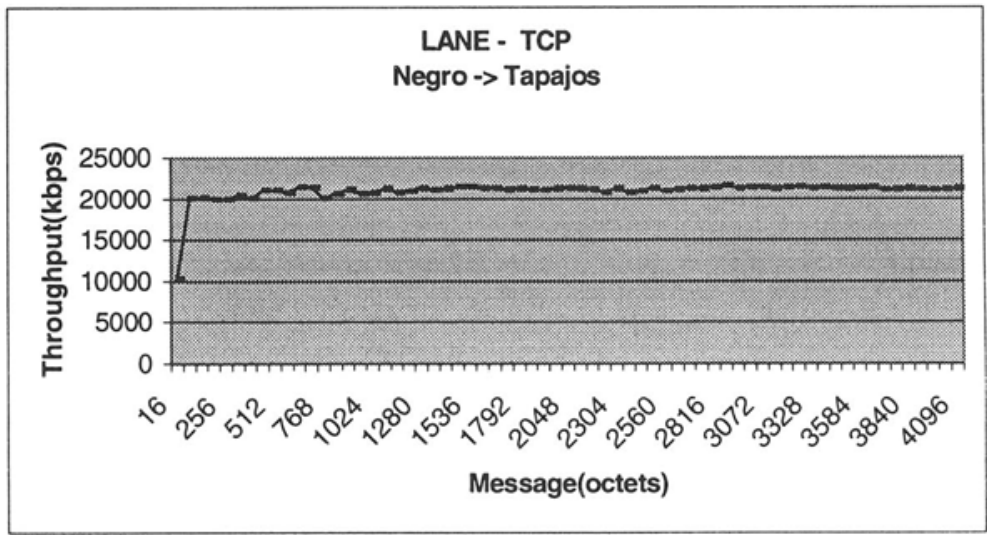

Figure 12: Throughput vs. message size

Table 4 shows the best values obtained in the Classical IP and LANE models.

Table 4: Maximum throughput obtained

\begin{tabular}{|l|c|c|c|c|}
\hline $\begin{array}{c}\text { Best } \\
\text { Results }\end{array}$ & $\begin{array}{c}\text { Theoretical } \\
\text { (Mbps) }\end{array}$ & $\begin{array}{c}\text { Measure } \\
\text { (Mbps) }\end{array}$ & $\begin{array}{c}(\%) \text { of } \\
\text { theoretical }\end{array}$ & $\begin{array}{c}(\%) \text { of } \\
\text { line rate }\end{array}$ \\
\hline Classical IP & 22.038 & 21.618 & 98.09 & 86.08 \\
\hline LANE & 22.038 & 21.579 & 97.91 & 86.08 \\
\hline
\end{tabular}

\section{CONCLUSIONS}

The Classical IP model presents a lower level of encapsulation than the LANE model, however the performance approached itself with a difference less than $0,1 \%$ among them. This was due to the workstations having 
sufficient software and hardware resources for operating in the rate of $25.600 \mathrm{Mbps}$, without significant delay of processing. In both models it could be observed that for small messages the performance was degraded considerably, this penalty in using the lower messages is that the protocols overhead become a significant percentage of the overall transmission rate.

The identified parameters in the testbeds made possible to determine the factors that influence the performance, among them we highlighted: application message size, socket buffer size and MTU. The capacity of processing of the stations The capacity of processing, of the interfaces and devices of software(device drivers) in spite of being important in the performance, presented sufficiency of resources for maintaining the network in the available speed .

\section{References}

[1] PARTRIDGE, Craig, "Gigabit networking". Addison-Wesley, April 1995.

[2] LAUBACH, M., J. Halpern, "Classical IP and ARP over ATM", RFC 2225, Newbridge Networks, April 1998.

[3] ATM Forum, "LAN Emulation Over ATM version 1.0" ATM Forum aflane-0021.000, Jan.1995.

[4] IBM 8285 Nways ATM Workgroup Switch, Installation and User's Guide, 1996.

[5] ITU-T Recommendation I.432.5 : "B-ISDN user-network interface Physical layer specification: 25,600 kbit/s operation", June 1997.

[6] ATM Forum, "Physical Interface Specification for $25.6 \mathrm{Mb} / \mathrm{s}$ over Twisted Pair Cable", af-phy-0040.000. Nov. 1995.

[7] ATM Forum, "User-Network Interface Version 3.1 Specification", Sep. 1994.

[8] ITU-T Recommendation I.363.5 - "B-ISDN ATM Adaptation Layer specification: Type 5 AAL". Aug. 1996.

[9] STALLINGS, William, "High-speed networks - TCP/IP and ATM design principles", Prentice-Hall, Inc., 1998.

[10] IEEE 802-3: "Carrier sense multiple access method", New York: IEEE, $1985 \mathrm{a}$.

[11] Hewlett-Packard Company, "Netperf: A network performance benchmark", revision 2.1, Information Network Division HewlettPackard Company, 1996.

[12] ANDRIKOPOULOS, T. Örs I et all, "TCP/IP throughput performance evaluation for ATM local area networks", Proceedings of 4th IFIP Workshop on Performance Modeling and Evaluation of ATM Networks, Ilkley, UK, July 1996. 